\title{
DISERTACIONES
}

ESTUDIOS

Para citar este artículo: Broitman, C., \& Rocamora, V. (2021). Ciencia y argumentos científicos en la controversia sobre la instalación del proyecto HidroAysén: normativa, debate y proceso. Anuario Electrónico de Estudios en Comunicación Social "Disertaciones", 14(2) 1-21. https://doi. org/10.12804/revistas.urosario.edu.co/disertaciones/a.10437

\section{CIENCIA Y ARGUMENTOS CIENTÍFICOS EN LA CONTROVERSIA SOBRE LA INSTALACIÓN DEL PROYECTO HIDROAYSÉN: NORMATIVA, DEBATE Y PROCESO}

Science and Scientific Arguments Regarding the Controversy about Execution of the Hidroaysén Project: Regulations, Discussions, and Procedures

Ciência e argumentos científicos na polêmica sobre a instalação do projeto HidroAysén: regulamentação, debate e processo

Claudio Broitman, Escuela de Periodismo, Universidad de Santiago de Chile (Chile) claudio.broitman@usach.cl

Verónica Rocamora, Escuela de Periodismo, Universidad de Santiago de Chile (Chile) veronica.rocamora@usach.cl

Recibido: 28 de marzo de 2021 Aprobado: 5 de mayo de 2021 Fecha de prepublicación: 1 de julio de 2021 


\section{RESUMEN}

La construcción de un gran proyecto hidroeléctrico en la Patagonia chilena produjo un debate que enfrentó los argumentos de promotores y opositores. Este artículo analiza la estrategia comunicacional de los principales actores de esta emblemática controversia y devela la forma en que ambos se adaptaron a la coyuntura, con diferente grado de flexibilidad, movilizando el discurso científico-técnico como marca de legitimación. Sin embargo, a medida que se mediatizó el conflicto y se definieron claramente los posicionamientos, se observaron cambios en la manera como se desplegaron los argumentos. Mientras que en el caso de los opositores se destaca una participación permanente de los científicos durante toda la controversia, no fue así con los promotores. Si bien estas voces expertas, que fueron reclutadas tempranamente por la empresa HidroAysén, se diluyeron durante el conflicto, se puede señalar que sus argumentos pervivieron a lo largo del debate.

Palabras clave: HidroAysén; controversia sociotécnica; científicos; debate; discurso.

\section{ABSTRACT}

The construction of a large hydroelectric project in the Chilean Patagonia sparked a discussion that faced the arguments of promoters and opponents. This article analyzes the communication strategy of main actors of this emblematic controversy, showing how both sides adapted themselves to the circumstances with different degrees of flexibility by using the scientific-technical discourse as a legitimization mark. However, as the conflict became covered by the media and positions were clearly defined, changes were observed in the way these arguments were presented. While in the case of the opponents, permanent participation of scientists is highlighted throughout the controversy; however, this was not the case with promoters. Although these expert voices, who were recruited early by the HidroAysén company, were diluted during the conflict, it can be noted that their arguments survived throughout the discussion.

Keywords: HidroAysén; socio-technical controversy; scientists; discussion; discourse.

\section{RESUMO}

A construção de um grande projeto hidrelétrico na Patagônia chilena produziu um debate que enfrentou os argumentos de promotores e opositores. Este artigo analisa a estratégia comunicacional dos principais atores desta emblemática polêmica, revelando a forma como ambos se adaptaram à situação, com diferentes graus de flexibilidade, mobilizando o discurso técnico-científico como marco de legitimação. No entanto, à medida que o conflito passou a ser mediado e as posições foram claramente definidas, foram observadas mudanças na forma como esses argumentos foram apresentados. Enquanto no caso dos adversários se destaca a participação permanente dos cientistas ao longo da polêmica, não foi o caso dos promotores. Embora essas vozes de especialistas, recrutadas precocemente pela empresa HidroAysén, tenham se diluído durante o conflito, pode-se notar que seus argumentos sobreviveram ao longo do debate.

Palavras-chave: HidroAysén; controvérsia sociotécnica; cientistas; debate; discurso. 


\section{DISERTACIONES}

ESTUDIOS

\section{Introducción}

El proyecto hidroeléctrico Aysén es un caso emblemático en el estudio de las controversias socioambientales en Chile, por diversas razones. Su simbolismo se debe no solo a la magnitud de lo proyectado, sino también a que se trata del primer conflicto de esa naturaleza capaz de movilizar la oposición masiva de la ciudadanía. Además, el debate suscitado se generó justo en el tránsito hacia una nueva normativa medioambiental, más estricta, que hizo más difícil la aprobación de grandes proyectos de impacto ambiental a partir de ese momento.

No habiendo presenciado hasta ese entonces conflictos socioambientales de esa magnitud, se produjo un nutrido debate en la opinión pública chilena. La confrontación de argumentos, discursos, intereses y dispositivos (columnas de opinión, pancartas, publicidad, etc.) alcanzó niveles jamás observados para una controversia de esta naturaleza en Chile, lo que dejó en evidencia tanto su alcance como una inusual heterogeneidad de voces, manifestadas de distintas maneras.

HidroAysén consistía, esencialmente, en la construcción de cinco megarrepresas, entre 2009 y 2022, en los lechos de los ríos Pascua y Baker, en la región de Aysén (Patagonia chilena), la más rica en recursos hidráulicos de ese país. Esto implicaba inundar 5910 hectáreas y levantar una línea de transmisión eléctrica de 1912 kilómetros hasta Santiago, la capital y principal consumidora de energía. El proyecto se constituyó en 2006, con la asociación de la empresa española Endesa ${ }^{1}(51 \%)$ y la chilena Colbún ${ }^{2}(49 \%)$. La capacidad de las cinco plantas en funcionamiento prometía llegar a 2750 megavatios y la energía debía incorporarse al sistema eléctrico central, que abastece a los principales centros de consumo energético del país. Según los estudios realizados por la empresa, el aporte energético representaría el $20 \%$ de la demanda. El costo del proyecto se estimó inicialmente en 3200 millones de dólares. ${ }^{3}$ La instalación de las mencionadas represas tenía reconocidas consecuencias negativas, como el desplazamiento de catorce familias, la expropiación de predios, la interrupción de caminos, el flujo de trabajadores, etc. Considerando lo anterior, HidroAysén implementó una estrategia de responsabilidad social empresarial, comprometiéndose a la construcción de infraestructura (caminos, puertos, escuelas, plazas, etc.) y a la capacitación de los habitantes mediante becas y donaciones, entre otras cosas. Asimismo, la empresa proponía reducir la dependencia a los combustibles fósiles de Chile, que era, al momento del proyecto, fuertemente tributario de la energía termoeléctrica (carbón, diésel o gas) e hidroeléctrica.

Sin embargo, las características específicas del territorio, en particular lo relativo a su aislamiento y a su valor patrimonial, fueron determinantes para que apareciera una oposición local y nacional al proyecto. En torno al Consejo de Defensa de la Patagonia (CDP) se articularon organizaciones de la sociedad civil, militantes ecológicos y habitantes. Se coordinaron fundamentalmente en redes sociales y lograron manifestaciones a lo largo de Chile bajo el eslogan de "Patagonia sin represas".

1 Esta empresa es a la vez propiedad de la multinacional Enel.

2 Propiedad de una de las familias más ricas de Chile, la familia Matte.

3 Se trata del costo inicialmente estimado para las cinco represas, pero que no calculaba el precio de la línea de transmisión eléctrica. 


\section{DISERTACIONES}

\section{ESTUDIOS}

La acción comunicacional del CDP contribuyó a una importante mediatización del conflicto (Broitman, 2017b). En una primera instancia, el Estado de Chile aprobó el Estudio de Impacto Ambiental (EIA) para la construcción de las represas, en mayo de 2011. Como reacción, miles de personas se manifestaron contrarias al proyecto a lo largo del país. Frente a esto, durante los meses que siguieron a esta aprobación, la empresa desplegó una agresiva campaña comunicacional favorable al proyecto en todos los medios de comunicación centrales del país. La mediatización del conflicto alcanzó su punto más alto en los años posteriores, hasta que en una segunda instancia fue rechazado bajo el Gobierno de Michelle Bachelet, en 2014 (tabla 1).

\section{Tabla 1. Hitos del proyecto}

\begin{tabular}{|l|l|}
\hline \multicolumn{1}{|c|}{ Año } & \multicolumn{1}{c|}{ Hito } \\
\hline 2004 & $\begin{array}{l}\text { La empresa Endesa inicia estudios preliminares para el levantamiento de la “línea de base” del proyecto } \\
\text { HidroAysén }\end{array}$ \\
\hline 2006 & Constitución de la empresa (51\% Endesa y 49\% Colbún) \\
\hline 2006 & Conformación del Consejo de Defensa de la Patagonia \\
\hline $2008-2010$ & $\begin{array}{l}\text { Proceso de evaluación ambiental (se presentan el estudio de impacto ambiental, tres informes consolidados } \\
\text { de solicitud de aclaraciones, rectificaciones o ampliaciones y tres adendas) }\end{array}$ \\
\hline 2011 & Aprobación inicial del estudio de impacto ambiental (solo represas) \\
\hline 2014 & Rechazo del proyecto en una segunda instancia por un comité de ministros \\
\hline 2017 & La empresa anuncia la cancelación del proyecto \\
\hline
\end{tabular}

\section{Fuente: elaboración propia.}

Este artículo analiza la controversia que se visibilizó en el espacio mediático, donde participaron mayoritariamente promotores y opositores al proyecto. Si bien su carácter científico-técnico fue fuertemente movilizado por la empresa, los detractores también se adaptaron a esta estrategia y respondieron en esos mismos términos. Además, en la elaboración burocrática del proyecto participaron científicos que también fueron adoptando posturas en virtud de su mediatización.

Tanto los promotores como los opositores desarrollaron estrategias para llegar a públicos definidos de acuerdo con sus objetivos. Así, mientras los boletines distribuidos por HidroAysén estaban dirigidos a los habitantes de la región, los avisos publicitarios del CDP estaban dirigidos a un público más amplio. Esto se debe a los objetivos de ambas partes: la primera pretendía establecer una comunicación con los habitantes; mientras que la segunda se oponía a un proyecto de gran envergadura, cuya discusión evolucionó de lo local a lo nacional. La comunicación profesional de HidroAysén también adquirió un alcance nacional a lo largo de los meses y en respuesta al curso del conflicto. 


\section{DISERTACIONES}

ESTUDIOS

Comunicación y controversias sociocientíficas en salud y medio ambiente

ISSN: 1856-9536

Doi: https://doi.org/10.12804/revistas.urosario.edu.co/disertaciones/a.10437

Volumen 14, Número 2 / Julio-diciembre 2021

Versión PDF para imprimir desde

http://revistas.urosario.edu.co/index.php/disertaciones

Nuestro enfoque toma como referencia principal el estudio de las controversias y señalan como primera coordenada los estudios sociales de la ciencia y tecnología (стs). Estos establecen la posibilidad de entender a los distintos actores de una controversia en un marco de equivalencia analítica. Tales condiciones permiten, a su vez, el despliegue de una cartografía (Akrich et al., 2006). Investigaciones posteriores que han dado continuidad a esta mirada de las controversias han planteado, por su parte, que la complejización de las cartografías, como todo mapa, puede ser una herramienta instrumentalizada políticamente. Sin embargo, el concurso de académicos y expertos de otros campos (como las comunicaciones) puede matizar ese efecto, ayudando a la comprensión de las cartografías y contribuyendo así al fortalecimiento de la democracia (Venturini et al., 2015).

Entendemos una controversia como "aquella que expresa un desacuerdo, una confrontación entre distintas racionalidades, entre diferentes concepciones de un mismo problema y del mundo social sobre el cual se despliega" (Badouard \& Mabi, 2015, p. 11). Las comunicaciones permiten abordar objetos poco observados en el despliegue de las controversias, como la circulación discursiva y de argumentos o la estructuración de un debate público sobre decisiones tecnocientíficas (p. 12). Así, se enfrentan en este campo en disputa todas las voces que, de una manera u otra, participan en la mediatización del conflicto. Sin perjuicio de que argumentos científico-técnicos hayan sido movilizados por los campos en contienda en la controversia sobre HidroAysén, como resultado del análisis se observa que hubo una heterogénea participación de los científicos en el mencionado debate.

\section{Controversias, expertos y riesgos}

Desde la década de 1960, la preocupación por los riesgos científico-técnicos y las consecuencias de la actividad industrial para las personas y su entorno generó una mayor conciencia medioambiental (Charvolin, 1993). Algunos fenómenos aislados, como los vertimientos de petróleo, la contaminación urbana, la gestión de residuos o los accidentes nucleares como el de Three Miles Island pasaron a entenderse desde una perspectiva ecológica más integral y a relacionarse con los modelos de desarrollo económico y productivo. La conciencia generalizada sobre los problemas medioambientales se manifestó a través de la cobertura mediática, con la institucionalización de estos problemas y, posteriormente, con la aparición de grupos ecologistas. A partir de entonces, el concepto de crisis medioambiental se instaló en la agenda pública como contrapunto al de desarrollo económico (Carson, 1962).

La centralidad de los expertos tomó relevancia para la sociología constructivista y con los "nuevos" problemas medioambientales. Trabajos pioneros como el de Dunlap y Catton (1979) desarrollaron interés por la construcción social de los problemas medioambientales, haciendo hincapié en que estos no surgen únicamente debido a las condiciones físicas, sino que también se configuran como resultado de las acciones de los distintos actores en el espacio público (Hannigan, 2006). El impulso inicial de analizar los problemas medioambientales más visibles, como la contaminación del aire o del agua, dio paso a "nuevos" problemas menos visibles, como los residuos tóxicos o el cambio climático. Estos nuevos problemas dependen de las mediciones e interpretaciones de los hallazgos de los científicos, sus análisis y propuestas de mejoras (Dunlap, 2015).

El rol de los expertos como voces autorizadas y legitimadas en los procesos de toma de decisiones de carácter público ha sido investigado desde diversas perspectivas, como los estudios sociales y culturales del riesgo, la sociología del conocimiento científico o los estudios cTs. En general, estos enfoques comparten algunos ejes, por ejemplo, el cuestionamiento a la visión tradicional de la ciencia como espacio autónomo y separado de la 


\section{DISERTACIONES}

ESTUDIOS

Comunicación y controversias sociocientificas en salud y medio ambiente

ISSN: 1856-9536

Doi: https://doi.org/10.12804/revistas.urosario.edu.co/disertaciones/a.10437

Volumen 14, Número 2 / Julio-diciembre 2021

Versión PDF para imprimir desde

http://revistas.urosario.edu.co/index.php/disertaciones

sociedad, la conciencia sobre la complejidad de los problemas científico-técnicos contemporáneos, la problematización de los diversos actores involucrados en los conflictos (incluidos los expertos) y la discusión sobre la legitimidad de la participación ciudadana en este tipo de conflictos.

Si bien el concepto de riesgo se ha utilizado ampliamente en diversos ámbitos, a partir de las visiones críticas de las décadas de 1960-1970 y de la inquietud generada por la energía nuclear, se abrió un campo de estudio sobre los riesgos de la tecnociencia, que tomó fuerza en la década de 1980 (López Cerezo \& Luján, 2000). El riesgo fue analizado desde una perspectiva sociológica-cultural por autores como Ulrich Beck, Anthony Giddens, Niklas Luhmann o Mary Douglas. Si bien inicialmente se convocó a los cientistas sociales en un intento por explicar los comportamientos "irracionales" de la ciudadanía frente a ciertos riesgos por factores sicológicos, sociales o culturales, estos autores terminaron problematizando a los mismos expertos como actores relevantes de los conflictos (Ramos Torre, 1999).

En el caso de los estudios cts y sus diversas escuelas, especialmente el Programa Fuerte, el Programa Empírico del Relativismo, el Social Construction of Technology y la Teoría del Actor Red, el rol de los expertos se ha analizado principalmente a partir del estudio de controversias sociotécnicas (García Palacios et al., 2001). Tal como se señaló, las controversias se refieren a los desacuerdos entre actores, los que pueden ser no solo humanos, sino incluir otros elementos biológicos y materiales que también se convierten en actantes (Venturini, 2010). Las visiones contrapuestas generadas en estas controversias se consideran un lugar privilegiado para el análisis de los procesos sociales y los mecanismos que utilizan los diversos actores que intervienen, ya sea científicos, activistas, empresarios, etc. Un aspecto relevante para los estudios cts es el fomento de la participación ciudadana en la toma decisiones en aquellos aspectos relacionados con la regulación de la ciencia y la tecnología (García Palacios et al., 2001).

En este punto se vuelve clave diferenciar quiénes son los actores legítimos para participar en una controversia. Si bien existen controversias científicas cerradas, en las que solo participan los expertos de un campo, diversos autores (Cuevas, 2008; Funtowitz \& Ravetz, 2000) han señalado que en las controversias complejas y cuyas consecuencias pueden afectar a públicos amplios o incluso a diversas generaciones, como los riesgos ecológicos, se vuelve imprescindible la participación de un público más amplio. En este caso, estaríamos frente a controversias sociocientíficas en las que participan no solo científicos-expertos, sino grupos que se consideran concernidos y donde no basta la evaluación de pares para cerrarlas o clausurarlas. Esta es la definición de controversia que tomamos como referencia para el caso de HidroAysén.

En los últimos años, este tipo de controversias sociocientíficas las han abordado investigaciones que ponen el acento en la relación-tensión de los expertos con grupos concernidos, como los tomadores de decisión, el público afectado, la prensa, entre otros (Rocamora et al., 2019; Young \& Matthews, 2007; Brüggemann et al., 2020; Barandarian, 2018). Un aspecto relevante para el análisis de este tipo de controversias son los mecanismos que utilizan los diversos actores para lograr su clausura o cierre. En este punto, los límites entre el conocimiento experto y el conocimiento "profano" se vuelven difusos, ya que los diversos actores movilizan tanto argumentos científicos como recursos retóricos o estrategias políticas para lograr posicionarse en la disputa. En este contexto, han sido objetos de interés las formas de circulación y apropiación del conocimiento científico por parte de la ciudadanía como estrategia de participación (Feng et al., 2020; Hernández \& Huepe, 2019; Sannazzaro, 2011). Por otra parte, se mantiene constante la pregunta que algunos autores (Collins \& Evans 2002; Collins et al., 2017) 


\section{DISERTACIONES}

ESTUDIOS

Comunicación y controversias sociocientificas en salud y medio ambiente

ISSN: 1856-9536

Doi: https://doi.org/10.12804/revistas.urosario.edu.co/disertaciones/a.10437

Volumen 14, Número 2 / Julio-diciembre 2021

Versión PDF para imprimir desde

http://revistas.urosario.edu.co/index.php/disertaciones

plantean respecto a legitimidad y extensión del conocimiento científico. La forma en que determinados instrumentos de base científico-técnica se han empleado para despolitizar los conflictos también han sido objeto de investigación (Buletti \& Ejderyan, 2020). En esa dirección, Baya-Laffite (2016) analiza cómo las evaluaciones de impacto ambiental (EIA) son instrumentos de gobernanza que trazan un guion que delimita no solo a los participantes, sino las reglas de participación y absorben las críticas opositoras. Así, los EIA serían "cajas negras" que las controversias contribuirían a abrir, por lo menos en parte.

Esta situación pudo observarse en la controversia de HidroAysén, ya que al abrirse la caja negra de su EIA, afloró el carácter fragmentario de sus mecanismos de evaluación y se evidenció la falta de un análisis integral. En ese sentido, y para lograr una perspectiva general, la ecología puede ser un aporte como disciplina, tanto desde un punto de vista discursivo como epistémico, que resulta fundamental para entender la trayectoria de los argumentos y el posicionamiento de los actores durante el transcurso del conflicto (Chateauraynaud, 2011).

\section{Metodologías para un corpus múltiple}

En el presente artículo se analizan una heterogeneidad de fuentes secundarias compiladas sobre la controversia descrita y colectadas en el espacio mediático: artículos de prensa nacional y local, publicaciones en línea, boletines emitidos por la empresa e insertos publicitarios del CDP. El periodo escogido es entre 2008 y 2013, ya que abarca el antes de la aprobación inicial del proyecto en 2011 y después de este año, cuando la controversia alcanza su cúspide, con una amplia movilización de la ciudadanía, lo que constituye un antecedente relevante para las actuales controversias medioambientales. A partir de los trabajos pioneros de Eliseo Verón (1981), tomamos como referencia una modalidad de discurso de tipo pedagógico, observando el despliegue de argumentos, la movilización de causalidades y el uso de marcas de legitimación (como el llamado a voces expertas o la puesta en evidencia de estudios). Asimismo, seguimos a Verón (1978) cuando advierte un "efecto ideológico", ahí donde el poder del discurso activa una creencia. A partir de estos elementos, estudiamos entonces las distintas configuraciones enunciativas de la manipulación (Breton, 2000), a la luz de la comunicación corporativa de la empresa, entre julio 2009 y diciembre 2013.

En el transcurso de la controversia, los actores desplegaron una panoplia argumentativa y una radicalización del léxico que puede situarse en el campo de la argumentación, en el de la manipulación o, incluso, en ambos. Esto último se relaciona con la estabilización de los enunciados y la atenuación de los conflictos de los discursos institucionales (Krieg-Planque \& Oger, 2010). En cuanto a la empresa, observamos en su comunicación profesional un esfuerzo de legitimación por medio de argumentaciones técnicas. En el caso de los opositores, ya que el objetivo principal era impedir el proyecto, la argumentación se despliega en diferentes direcciones. En consecuencia, los documentos analizados son bastante homogéneos para el caso de la empresa y más bien híbridos en relación con los opositores.

El corpus relativo a la empresa se divide en dos partes: la primera se refiere a un total de ocho boletines distribuidos en la región (julio de 2009-junio de 2011), y la segunda, las cuatro últimas publicaciones distribuidas en el mismo territorio (noviembre de 2011-diciembre de 2013). Asimismo, también analizamos los contenidos comunicacionales difundidos por el CDP entre agosto de 2008 y diciembre de 2013. Se trata, fundamentalmente, de 


\section{DISERTACIONES}

ESTUDIOS

Comunicación y controversias sociocientificas en salud y medio ambiente

ISSN: 1856-9536

Doi: https://doi.org/10.12804/revistas.urosario.edu.co/disertaciones/a.10437

Volumen 14, Número 2 / Julio-diciembre 2021

Versión PDF para imprimir desde

http://revistas.urosario.edu.co/index.php/disertaciones

35 avisos publicitarios publicados en prensa escrita local y nacional, destinados a la formación de una opinión pública opuesta a la realización de las represas.

El corpus también está compuesto por una revisión de documentos técnicos para la reconstrucción de los sucesos, así como para su comprensión crítica. En virtud de dicha revisión, identificamos la producción de artículos científicos y de ciencia regulatoria (Jasanoff, 2009) que también se analizaron con miras a la cartografía enunciada. Finalmente, realizamos una búsqueda en profundidad de los artículos científicos y producciones culturales de expertos que se opusieron públicamente al proyecto HidroAysén durante el año de mayor intensidad de la controversia (2010). Dicha búsqueda se apoyó en la sistematización de categorías emergentes emanadas de trabajos anteriores (Broitman, 2017a).

\section{El proyecto como una oportunidad para los científicos}

En 2004, Endesa inició los estudios preliminares para el Proyecto Hidroeléctrico Aysén, lo que se denomina técnicamente el establecimiento de una línea de base. Este proceso, que no llamó la atención de los medios de comunicación, era un requisito para poder desarrollar posteriormente el ElA y llevarlo a cabo. La empresa, entonces, realizó una licitación privada para financiar un compendio de estudios en el que participaron expertos de diversos ámbitos. La empresa contrató los servicios de varias universidades chilenas y de un consultor local (Centro de Ecología Aplicada), cada uno según sus áreas de especialización. La Universidad de Chile realizó, por un lado, el estudio de los suelos, el clima, la meteorología, la hidrología, la geología y la geomorfología; por otro, el estudio de las superficies de riesgo. La Pontificia Universidad Católica de Chile hizo un estudio sobre la población, los aspectos socioeconómicos, la calidad de vida y las actividades turísticas y otro sobre la propiedad de la tierra, la infraestructura y el equipamiento. La Universidad Austral realizó estudios sobre la vegetación y la flora terrestre. La Universidad de Concepción llevó a cabo un estudio sobre la fauna terrestre. La Universidad de Valparaíso realizó estudios de oceanografía, flora y fauna marina y estuarina. La Universidad Central desarrolló estudios de paisaje, ordenamiento territorial y áreas protegidas. Por último, la Universidad Bolivariana evaluó el patrimonio cultural. Entre tanto, el Centro de Ecología Aplicada realizó un estudio de flora y fauna acuática, calidad del agua y caudal ecológico. Según la empresa, los estudios abarcaron un territorio de casi 300000 hectáreas durante dos años.

El EIA fue elaborado por tres consultoras (Poch, EPS y Sweco) a partir de la información científica recabada durante los estudios preliminares. Esta información, propiedad de la empresa, se presentó de forma editada en el EIA para ser entregada a los servicios de evaluación, en agosto de 2008. La Ley 19.300 indicaba que los servicios del Estado debían evaluar el impacto en sus ámbitos de competencia. Por ejemplo, el Servicio Forestal Nacional, dependiente del Ministerio de Agricultura, analiza el impacto del proyecto en los bosques. En este marco, la Ley 19.300 favoreció una evaluación por sectores: sin comunicación entre los servicios era imposible una visión global de los estudios realizados.

La ley exigía que si no había suficientes estudios científicos para evaluar el impacto de un proyecto, era responsabilidad del promotor proporcionar los estudios necesarios (los estudios preliminares) para que los servicios pudieran desempeñar sus funciones. En este marco, la solicitud de aclaraciones y complementos a los informes presentados 


\section{DISERTACIONES}

ESTUDIOS

Comunicación y controversias sociocientificas en salud y medio ambiente

ISSN: $1856-9536$

Doi: https://doi.org/10.12804/revistas.urosario.edu.co/disertaciones/a.10437

Volumen 14, Número 2 / Julio-diciembre 2021

Versión PDF para imprimir desde

http://revistas.urosario.edu.co/index.php/disertaciones

es responsabilidad de los servicios públicos. Excepcionalmente, estos servicios podrían realizar estudios independientes, pero la ley no dotaba a los servicios de medios específicos para ello. ${ }^{4}$

En el caso particular del Proyecto Hidroeléctrico Aysén, se produjo una situación nueva, debido a la magnitud del proyecto y a la carencia de estudios científicos en el territorio estudiado. Salvo algunas excepciones, dichos estudios no eran publicaciones científicas, sino encargos de la empresa para el proyecto, y realizados por científicos (censos, base de datos, etc.). Los informes tenían formatos heterogéneos: aunque la ley exigía al proponente incluir una línea de base en su EIA, no especificaba en qué consistían. Así, pues, la heterogeneidad de los estudios preliminares se debe a las condiciones de producción; su calidad científica depende de los autores y de los enfoques (metodología, condiciones de campo, etc.), ya que no se sometieron a una revisión por pares ni a una evaluación por parte de la empresa, por ejemplo, a través de consultores. Así, nos parece relevante señalar que en la introducción del resumen del EIA (2008) de HidroAysén, la empresa presentó el levantamiento de la línea de base como:

El presente EIA está basado en una caracterización medioambiental que abarcó estudios en cerca de 300000 hectáreas, un área varias veces superior a la superficie de obras y embalses del proyecto. Durante un prolongado espacio de tiempo, más de 2 años según corresponda, prestigiosas universidades y centros de investigación realizaron diversas campañas en terreno, generando una gran cantidad de información nueva sobre la Región de Aysén.

De esta manera, como estrategia argumentativa, se busca trasladar al documento la reputación concedida a las instituciones científicas. Los datos recogidos por los 370 investigadores ${ }^{5}$ que participaron en el levantamiento de las líneas de base forman parte de la caracterización descrita. Estos datos, que eran propiedad de la empresa durante un periodo determinado (los contratos privados establecían diferentes criterios para los proveedores de servicios), han dado lugar en ocasiones a publicaciones científicas con el consentimiento de la empresa (Arenas \& Osses, 2012; Ramírez et al., 2012). Por consiguiente, la asignación de fondos para estudiar una región desconocida era un medio de financiar la actividad científica.

La información recogida en los estudios preliminares también la difundió la empresa como parte de su estrategia de "comunicación e información" en la región. Uno de los argumentos centrales de dicha difusión fue la exigencia de absoluta transparencia y total apertura en cuanto a la información recogida. En este contexto, la EIA afirmaba en su resumen que:

La fase de estudios del proyecto se ha abordado de manera innovadora en las diferentes dimensiones que

lo componen. Es así que una de sus principales características ha sido establecer una comunicación anticipada proactiva, abierta y transparente desde el inicio de los estudios, con el objetivo de informar oportunamente a las comunidades, líderes de opinión y autoridades locales, regionales y nacionales, acerca de los avances del proyecto y los componentes medioambientales estudiados. (EIA, 2008)

4 Esta carencia fue resuelta por la Ley 20.417 (2010), al establecer la creación de un Ministerio del Medio Ambiente que tiene como una de sus funciones la realización de estudios preliminares necesarios para la evaluación de proyectos que impacten el medio ambiente.

5 Cifra publicada por la empresa en el EIA. 


\section{DISERTACIONES}

ESTUDIOS

En cuanto al levantamiento de la línea de base, esta voluntad se materializó con dos iniciativas. La primera fue la instalación de "casas abiertas", que eran centros de difusión permanentes e itinerantes en la región para que los habitantes pudieran acceder a toda la información recogida, incluidos los estudios preliminares; pero sin edición ${ }^{6}$ (Tironi \& Barandiarán, 2014). La segunda consistió en reuniones entre los actores locales y algunos de los científicos que habían participado en los estudios preliminares. Dichos encuentros se realizaron en enero de 2008, en Coyhaique y Cochrane, las dos principales ciudades afectadas por el proyecto. En estas reuniones, los científicos que habían ejecutado los estudios preliminares presentaron sus resultados a los habitantes, representantes políticos, empresas locales, sindicatos, etc. En las reuniones participaron representantes de la Pontificia Universidad Católica de Chile, la Universidad Austral, la Universidad de Valparaíso y el Centro de Ecología Aplicada. Al participar voluntariamente en estas actividades, los científicos reivindicaron el trabajo de los estudios preliminares.

Estas reuniones científicas fueron organizadas conjuntamente por HidroAysén y el Centro de Investigación de Ecosistemas de la Patagonia (CIEP), recientemente fundado (2005), y que validó públicamente la metodología utilizada en los estudios preliminares. En esa ocasión, el director del cIEp explicó a un periódico regional por qué el centro estaba asociado a una empresa:

Los procesos validados por la metodología científica establecen sin lugar a dudas cuál es el inventario actual de la flora y la fauna en el río Baker y sus alrededores, forman parte de la realización de estudios preliminares para un estudio medioambiental. El objetivo es también determinar el estado de las aguas, las costas, los lechos y la atmósfera del río y sus alrededores. Si la realización de estos estudios ha sido encargada al CIEP y/o a las Universidades asociadas al Centro, esta es prácticamente la situación ideal, lo que implica que estos estudios previos sean realizados por científicos de reconocida excelencia y probidad.

(Citado en Broitman, 2017b, p. 42)

Posteriormente, el cIEP se distanció del proyecto hidroeléctrico Aysén, ya que no hay constancia de su participación en el EIA. En síntesis, más allá del ejemplo del CIEP, se puede observar cómo la empresa incorporó a científicos como parte de su estrategia de legitimación desde el principio, apostando al reclutamiento de voces expertas favorables al proyecto.

\section{Las voces disidentes}

Algunos científicos expresaron su oposición al proyecto HidroAysén, particularmente en el Centro de Ciencias Ambientales EuLA-Chile de la Universidad de Concepción. ${ }^{7}$ HidroAysén invitó al Centro eULA a participar en los estudios preliminares, pero el centro declinó la invitación por no estar de acuerdo con los términos de referencia propuestos en el contrato: los investigadores del centro se oponían especialmente a participar solo en el levantamiento de línea de base del proyecto HidroAysén. También querían participar en la EIA, pero la empresa no aceptó esta modalidad.

6 La caracterización ambiental completa no volvió a estar disponible para el público.

7 El Centro eULA participó directamente en la fundación del CIEP. 


\section{DISERTACIONES}

ESTUDIOS

Comunicación y controversias sociocientificas en salud y medio ambiente

ISSN: 1856-9536

Doi: https://doi.org/10.12804/revistas.urosario.edu.co/disertaciones/a.10437

Volumen 14, Número 2 / Julio-diciembre 2021

Versión PDF para imprimir desde

http://revistas.urosario.edu.co/index.php/disertaciones

Hubo redes científicas que se configuraron durante el periodo en que se discutió el proyecto HidroAysén. Un ejemplo es la colaboración entre científicos del Centro eULA e investigadores internacionales.

Goodwin et al. (2006) ${ }^{8}$ publicaron sobre las consecuencias ecológicas y sociales de las grandes represas del mundo. Consideran que su gestión es un problema científico relativamente nuevo en comparación con el tiempo que se necesita para detectar y comprender sus consecuencias. Analizan el desarrollo hidroeléctrico en la Patagonia, considerando el equilibrio entre el contexto energético, la belleza escénica (o paisajística) de la región, su ecología y la industria del salmón. Los autores se preguntan si las represas son justificables, dadas las prioridades sociales y reflexionan sobre los daños al ecosistema y, en particular, a la biodiversidad.

Otro caso es el de Winemiller et al. (2016), quienes piden una planificación hidroeléctrica más sofisticada y holística. El artículo publicado en Science argumenta que los protocolos para ese desarrollo suelen desconocer los impactos acumulativos en la hidrología y los servicios de los ecosistemas en las cuencas. Proponen ir más allá de los impactos locales de los proyectos, pensando en las posibles sinergias con otros desarrollos en las cuencas.

Volviendo a los científicos del Centro EULA, Wright et al. (2008) escribieron sobre la importancia de planificar el agua como recurso, en términos socioterritoriales y de conservación de la biodiversidad. Desde una perspectiva internacional, cuestionaron los riesgos asociados a la construcción de grandes represas. Por ejemplo, consideraron la bien documentada influencia de la represa de Libby en la población de esturión blanco del río Kootenai (Montana, Estados Unidos). El impacto del proyecto de ingeniería en el flujo y la temperatura del agua destruye esta especie. Aunque los autores admitieron que HidroAysén no generaría este tipo de problemas, porque las represas serían reguladas por los lagos, sugirieron que nuevos problemas podrían influir en la biodiversidad de la Patagonia, considerando que era una región muy poco estudiada en ese momento.

Wright et al. (2009)9 describieron una colaboración entre la Universidad de Concepción y la Universidad de Michigan, en respuesta a una convocatoria de un curso interdisciplinario que problematiza el desarrollo sostenible. El tema principal de esta iniciativa fueron las necesidades de electricidad de Chile y el proyecto hidroeléctrico de Aysén en la Patagonia. Integrando cuestiones sociales sobre el agua, la energía y la conservación de la biodiversidad, el curso proponía reflexionar sobre las consecuencias de desarrollos similares, la sostenibilidad de las decisiones a largo plazo y las soluciones alternativas a los problemas propuestos a lo largo del curso.

Dusaillant et al. (2010) ${ }^{10}$ se refirieron al fenómeno del Glacial-Lake Outburst Flood (GLOF). Se trata de eventos de drenaje gigantesco de los lagos glaciares hacia los ríos, capaces de aumentar sus caudales de forma aguda e imprevisible. El artículo describía cinco sucesos ocurridos en los últimos meses - al momento de su publicaciónen el lago Cachet 2, los mayores jamás registrados (desde 1963), lo que sugiere una relación con el calentamiento global. Los investigadores criticaron la metodología propuesta por HidroAysén en el EIA para calcular los riesgos asociados a los GLOF y cuestionaron en particular la capacidad de respuesta ante un evento de este tipo.

8 Óscar Parra (EULA/CIEP) y Claudio Meier (Universidad de Concepción/CIEP) participaron también en este trabajo que se publicó en la revista Hydroinformatics.

9 Evelyn Habit y Óscar Parra, también del Centro EULA, son autores de este trabajo.

10 Claudio Meier es uno de los autores de este artículo. 


\section{DISERTACIONES}

ESTUDIOS

Piper y Fajardo (2011a), científicos del CIEP, en respuesta a una carta publicada por Vince (2010) en la revista Science, afirmaron que las represas tienen un efecto sobre el calentamiento global, ya que las áreas que inundan la biomasa forestal densa (como la propuesta de HidroAysén en su EIA) generan emisiones de dióxido de carbono particularmente altas. También señalaron que los ríos de la Patagonia se alimentan de los glaciares, un recurso no renovable. Por lo tanto, el proyecto no sería económicamente viable, ya que esta condición limitaría la producción hidroeléctrica a mediano y largo plazo. Por último, refutaron el pronóstico de que las sequías serían frecuentes en el futuro en la zona central de Chile y que la Patagonia no se vería afectada por este fenómeno, argumentando que las predicciones para Sudamérica no eran fiables, ya que a menudo carecían de modelos.

En otro artículo, los mismos investigadores argumentaron que no eran fiables los planes de reforestación propuestos por la empresa (Piper \& Fajardo, 2011b). Señalaron que al no disponer de suficiente información sobre la región, los estudios se basaban en estadísticas de otras regiones. Así, el plan proponía la introducción de especies foráneas, lo que exigía la eliminación de las especies competidoras de estas de las plantaciones. Consideraron que la reforestación propuesta por el proyecto hidroeléctrico Aysén no incluía la colaboración de científicos, ingenieros y población local en la fase de desarrollo (World Commission on Dams, 2000).

Además, científicos y expertos opuestos al proyecto dieron a conocer sus posturas, mediante diversos medios de comunicación alternativos a las publicaciones científicas tradicionales. Dichas críticas apuntaban o se construyeron con base en el EIA. Pocos días después de la aprobación de las represas, Claudio Meier (2011) publicó un texto en internet donde criticaba con argumentos técnicos el Proyecto Hidroeléctrico Aysén. En el texto titulado "Hidroelectricidad realmente sustentable para Chile", el ingeniero hidráulico se declaró favorable a los "proyectos grandes de hidroelectricidad sustentable". Sin embargo, señala la existencia de "múltiples falencias" en la normativa legal chilena y en el propio proyecto HidroAysén. Basándose en un análisis histórico de la hidroelectricidad y en los elementos movilizados en el EIA de su campo de especialización (ecología fluvial), condenó el proyecto hidroeléctrico de Aysén como "una obscenidad ambiental, social y económica".

Roberto Román (2011), profesor de la Universidad de Chile y especialista en energía solar, publicó en la web de la Facultad de Ingeniería de su universidad un artículo titulado "Diez razones por las que HidroAysén no es necesaria ni conveniente para Chile". El ingeniero mecánico argumentó que la necesidad de duplicar la producción de energía es "un mito fabricado por la industria", haciendo hincapié en el desarrollo de las energías renovables no convencionales cerca de los centros de consumo, tomando como ejemplo el potencial de energía solar del desierto de Atacama como fuente de abastecimiento para la minería de este territorio. También propuso la implementación de una estrategia de eficiencia, seguridad energética y diversificación de los productores de energía, ya que HidroAysén favoreció la consolidación de un oligopolio en el sector, dejando poco espacio para proyectos que pudieran diversificar la producción de energía. 


\section{DISERTACIONES}

ESTUDIOS

\section{El dominio técnico de la empresa en la aprobación de proyectos}

En agosto de 2008 se inició el proceso de evaluación ambiental, ${ }^{11}$ en virtud de la presentación de un EIA entregado por la empresa, según lo que mandataba la ley. ${ }^{12} \mathrm{El}$ procedimiento consistía en que la empresa presentaba el EIA y los servicios públicos lo revisaban posteriormente. Dicha tarea era responsabilidad de la Comisión Regional de Medio Ambiente, que, a su vez, emitió luego tres documentos denominados Informe consolidado de solicitud de aclaraciones, rectificaciones o ampliaciones (ICSARA), en respuesta al mencionado EIA. Además, las respuestas de la empresa (a través de sus consultores) se pueden encontrar en otros informes, las adendas. Entre 2008 y 2010 la empresa presentó tres adendas ${ }^{13}$ a los tres ICSARA.

HidroAysén tuvo un modus operandi que ya había sometido a prueba, en virtud de los grandes proyectos ejecutados por las empresas propietarias del proyecto (Endesa y Colbún) y consolidado por el acuerdo previo del grupo de promotores del proyecto ${ }^{14}$ para llevar a cabo la operación. La estrategia aplicada sigue así una lógica interna, herencia de una cultura empresarial probada durante décadas con varios proyectos. Endesa realizó sus estudios de ingeniería a través de su filial Ingendesa, el grupo de expertos con más experiencia en la construcción de proyectos hidroeléctricos en Chile. Asimismo, la validación de los estudios preliminares se llevó a cabo en las universidades más prestigiosas. Además, los consultores contratados también fueron especialistas en su campo, lo que garantizó la aprobación de los proyectos en un marco jurídico (Ley 19.300) que no estaba diseñado para rechazar los proyectos, sino que para mejorarlos (Tecklin et al., 2011).

\section{El discurso científico-técnico de HidroAysén en tensión}

Sin embargo, la estrategia de HidroAysén no se adaptó a los acontecimientos externos (especialmente mediáticos) que se produjeron durante la cobertura del conflicto, sobre todo en lo relativo a la novedad de las acciones publicitarias de los grupos opositores al proyecto.

Si bien es posible observar una voluntad de la empresa para discutir sobre los temas controversiales que están en juego en el proyecto, el discurso nunca transgrede los márgenes de la comunicación social empresarial. Por ejemplo, en los boletines analizados se da más espacio e importancia a las acciones de la empresa en la región que al debate sobre la implantación de las represas hidroeléctricas.

11 Uno de los aspectos polémicos fue que este estudio solo abarcaba la construcción de las centrales hidroeléctricas, pero no la instalación de la línea de transmisión eléctrica.

12 Si bien HidroAysén presentó su EIA bajo la Ley 19.300 de Medio Ambiente, la nueva ley del sector (20.417) establece el mismo mandato para los promotores de los proyectos. Sin embargo, una diferencia fundamental es que la nueva ley, al contemplar una Superintendencia de Medio Ambiente, puede solicitar nuevos estudios independientes para la evaluación de los proyectos, y no necesariamente estudios financiados por el promotor.

13 Para más información, véase Adenda (2008).

14 Este grupo de promotores, integrado por empresarios, profesionales, representantes y ciudadanos será analizado más adelante. 


\section{DISERTACIONES}

ESTUDIOS

Comunicación y controversias sociocientificas en salud y medio ambiente

ISSN: 1856-9536

Doi: https://doi.org/10.12804/revistas.urosario.edu.co/disertaciones/a.10437

Volumen 14, Número 2 / Julio-diciembre 2021

Versión PDF para imprimir desde

http://revistas.urosario.edu.co/index.php/disertaciones

A menudo, la voluntad permanente de la empresa de argumentar a favor del proyecto choca con la necesidad de crear vínculos con los habitantes. Esta voluntad se ve confirmada por la presencia de temas relevantes para el proyecto y el uso de otros argumentos, que se observan regularmente en el corpus analizado y que son importantes para los editores de los boletines, como la contribución de la energía hidroeléctrica a la reducción de las emisiones de dióxido de carbono.

El objetivo de los boletines es comunicar las acciones de la empresa en la región, reforzar los vínculos entre la empresa y los habitantes y difundir información relacionada con la producción de energía. El énfasis en las figuras enunciativas y el uso de estudios, figuras y conceptos en el planteamiento argumentativo atestiguan la voluntad permanente de argumentar en todos los contenidos que no están relacionados con la responsabilidad social empresarial. Esto también se expresa mediante el empleo de contenidos técnicos en la comunicación profesional dirigida a los habitantes de la región. El análisis muestra que esta voluntad se ve debilitada cuando los medios de comunicación cubren el conflicto.

El despliegue de contenidos técnicos puede verse en los boletines. En el quinto ejemplar de mayo de 2010, bajo el título “¿Sabías que?”, el enunciador afirma: “HidroAysén producirá anualmente $18.430 \mathrm{Gw} / \mathrm{h} .{ }^{15}$ Esta cifra representa el $77 \%$ de la producción hidroeléctrica total de Chile en 2008 y el $35 \%$ del consumo total de electricidad de nuestro país en el mismo año". El vínculo declarado entre la producción y el consumo, expresado en cifras, establece una correspondencia en el ámbito técnico. Asimismo, la producción prevista para el proyecto se establece a partir de estudios independientes realizados por la empresa, lo que da a la declaración una impronta científico-técnica.

La sección "¿Sabías que...?” se presenta al lector como una cápsula aislada de todos los textos que se expresan en los boletines. Sin embargo, es necesario, ya que recuerda al lector la propia naturaleza del producto, es decir, un proyecto de desarrollo hidroeléctrico. Este epígrafe, que permite situar el enunciado de tipo científico en un contexto nacional (el consumo total de electricidad de nuestro país), resalta un aspecto central que a posteriori se convierte en la fuente misma del conflicto y también es importante para sus productores. En efecto, la estrategia de comunicación de los grupos que se oponen al proyecto obliga a la empresa a cambiar sus acciones de comunicación en un intento por destacar los aspectos técnicos positivos del proyecto a escala de todos los habitantes de Chile.

La empresa se vio presionada por el fenómeno de la mediatización, alimentado principalmente por la acción de grupos opositores y la decisión de la Comisión de Evaluación Ambiental de aprobar la construcción de las cinco represas en la Patagonia chilena. El reto ya no era argumentar en el ámbito regional, sino convencer a todo el país de que el proyecto era necesario y positivo. Así, cambió la composición de la construcción discursiva. Aunque las configuraciones discursivas descritas persistieron, se identificaron algunos elementos nuevos. Se materializaron sobre todo en la irrupción de nuevos temas (como las energías renovables no convencionales) y en la movilización de un léxico diferente.

El noveno boletín, publicado en noviembre de 2011, es una respuesta a la mencionada tensión socioeconómica. En la cabecera no hay ningún representante de la empresa, sino retratos de cuatro habitantes de la zona (dos mujeres y dos hombres), ya sea en plena naturaleza o con atributos de la región (un acordeón, un sombrero, etc.).

15 Gigawatt por hora, escrito como símbolos en el original. 


\section{DISERTACIONES}

ESTUDIOS

Comunicación y controversias sociocientificas en salud y medio ambiente

ISSN: 1856-9536

Doi: https://doi.org/10.12804/revistas.urosario.edu.co/disertaciones/a.10437

Volumen 14, Número 2 / Julio-diciembre 2021

Versión PDF para imprimir desde

http://revistas.urosario.edu.co/index.php/disertaciones

El titular dice: "HidroAysén reducirá la factura eléctrica en un $50 \%$ ". Más adelante, en los recuadros, hay algunas declaraciones firmadas por los residentes. Por ejemplo: "Recuerdo que cuando se construyó la Carretera Austral, los habitantes dijeron que al abrir las carreteras estaban destruyendo la Patagonia. Hoy piden al Estado que asfalte las carreteras. Yo veo lo mismo con HidroAysén" (Loreto Higuera, residente de Puerto Tranquilo, citado en HidroAysén, 2009).

De esta forma, a partir del análisis se puede observar cómo la empresa va adaptando su estrategia comunicacional a medida que avanza el conflicto: cambios de escala regional-nacional, movilización de nuevas voces para legitimar argumentos (habitantes) o nuevos temas, como el de desarrollo sostenible. Sin perjuicio de lo anterior, observamos también que la empresa mantuvo una línea argumental regular centrada en los costos y beneficios del desarrollo durante todo el periodo observado. Cabe destacar que en el corpus analizado no se aprecia la participación directa de científicos o expertos, sino que son los mismos representantes de la empresa quienes movilizan el discurso científico-técnico.

\section{Los opositores también movilizan argumentos científico-técnicos}

El análisis muestra que la estrategia comunicacional del CDP también tiene un cambio de escala, que no responde solo al conflicto ni tampoco a la discusión sobre la protección del patrimonio natural. "HidroAysén se ha convertido en todo lo que no queremos como chilenos", afirmó un periodista de los opositores entrevistado para esta investigación. Así, la imagen construida de la empresa es la de "las" empresas. La situación social efervescente que se vivía en el país (y en el mundo) en 2011, ligada a las manifestaciones por la educación pública, también contribuyó al éxito de esta construcción. Así, la primera parte de la campaña, que buscó santuarizar la Patagonia y mostrar una imagen de la empresa como su destructora, fue bien recibida por algunos sectores en este contexto (Broitman, 2017b).

Esta campaña publicitaria movilizó fuertemente el tema medioambiental, pero a partir de categorías no utilizadas por la empresa, como la conservación del patrimonio natural o el paisaje. La publicidad desplegada por el CDP utilizó una comunicación persuasiva (Breton, 2000) e intentó llevar el conflicto a escala nacional. Esta ampliación implicó un cambio de escala basado en la creación de una audiencia (Lezaun \& Soneryd, 2007), desarrollada a partir de encuestas y convocatorias populares para manifestarse a lo largo de Chile y que es particularmente sensible a las críticas al poder gobernante.

La forma en que el CDP representó a la naturaleza (la Patagonia) también fue cambiando, dependiendo de la coyuntura de los acontecimientos. En una segunda etapa, la campaña publicitaria pasó de la crítica de las empresas a la de las instituciones, divulgando las gestiones relacionadas con el EIA.

La lógica de los detractores se traduce en una comunicación militante y plástica con un único objetivo: impedir la realización del proyecto. A diferencia de la empresa, esta adaptabilidad les permitió asumir diferentes roles, construidos a partir de las evidencias recogidas en el espacio mediático o a partir de la configuración de una narrativa (la santuarización de la naturaleza o la escenificación del conflicto). Así, los detractores adaptaron su publicidad según el curso de los acontecimientos, incluso resignificando los argumentos de los promotores del proyecto.

La estrategia comunicacional de los opositores, sensible a todo aquello que circula en el espacio mediático, admite entonces otras configuraciones enunciativas y otros argumentos: se denuncia a la empresa en nombre de 


\section{DISERTACIONES}

ESTUDIOS

Comunicación y controversias sociocientificas en salud y medio ambiente

ISSN: $1856-9536$

Doi: https://doi.org/10.12804/revistas.urosario.edu.co/disertaciones/a.10437

Volumen 14, Número 2 / Julio-diciembre 2021

Versión PDF para imprimir desde

http://revistas.urosario.edu.co/index.php/disertaciones

una crítica a las lógicas de la producción industrial de energía y a partir de un llamado a un consumo energético responsable. Emplean un léxico radical y llevan una panoplia argumentativa que tiende a la denuncia, la indignación y la representación.

En octubre de 2010 apareció un inserto publicitario del CDP en el diario La Tercera, que publicó "El maquillaje verde de HidroAysén. La realidad frente a la propaganda”. En la parte superior de la página, un paisaje patagónico atravesado por torres de alta tensión. A la derecha, una enorme cara del Joker de Batman maquillada de verde. Como la presencia de este personaje no se explica en absoluto en el documento, el lector asume que se trata de una amalgama cognitiva del maquillaje que utiliza el Joker, amalgama que apela a la herencia cultural de los coenunciadores: el Joker es un villano que se maquilla para ocultar su cara quemada.

El enunciador discute en un apartado titulado "HidroAysén perjudicaría el valor ecológico y turístico de la región de Aysén":

Un estudio de la Universidad de Chile demostró que durante los doce años de construcción de las centra-

les hidroeléctricas de HidroAysén, la región de Aysén dejaría de recibir al menos 400 millones de dólares por el impacto negativo en el turismo. Así, se perderían 4.000 puestos de trabajo, mientras que las presas solo crearían 140 empleos. Un proyecto de esta envergadura destruiría o degradaría un paisaje de alto valor ecológico, perjudicando de forma irreversible el altísimo potencial de turismo sostenible que está empezando a desarrollarse en la región. (CDP, 2011a)

Basándose en un estudio dotado de legitimidad científica (la Universidad de Chile), el enunciador evoca un tema central ya utilizado por los promotores del proyecto: el empleo. Por otro lado, discute el alcance y la calidad del empleo que proporcionarían las represas, y lo relaciona con las consecuencias del proyecto hidroeléctrico en la región. Aquí vemos cómo los habitantes (a través del empleo) se sitúan en el centro del argumento sobre el impacto del proyecto. El argumento es que el turismo sustentable (como tema que integra cuestiones energéticas y medioambientales) es potencialmente un mejor proveedor de puestos de trabajo que las represas. Además, también se moviliza la noción de costo de oportunidad, ya que el argumento sugiere que evitar la construcción del proyecto dejaría espacio para el desarrollo de este turismo. La estrategia comunicacional del cDP parece, pues, sensible al debate público.

En otro inserto publicado en el diario El Mercurio (diciembre de 2009) que aboga por la instalación de métodos de producción de energía limpia para Chile, el enunciador señala en un recuadro:

Utilizando fuentes de energía renovables no convencionales y aplicando una política de eficiencia energética,

es posible eliminar el proyecto HidroAysén, la pesadilla nuclear y la producción de carbón. De este modo,

se evitan los gravísimos impactos negativos de las tecnologías contaminantes y destructivas: ecológicos,

sociales, económicos y culturales. (CDP, 2011b)

El redactor describe las fuentes de energía no renovables como contaminantes y destructivas. A pesar de que no explica la razón de los gravísimos impactos que denuncia, pretende demonizar cualquier fuente de energía que no sea la de las energías renovables no convencionales.

El objetivo de la inserción es proponer alternativas al proyecto. Los medios tradicionales de producción se presentan como indeseables; mientras que las energías renovables no convencionales serían el mejor camino para el desarrollo de la electricidad. Se observa una demonización de la energía nuclear como alternativa eléctrica para Chile, expresada en la palabra pesadilla. En el mismo inserto se critica este tipo de producción de energía: 


\section{DISERTACIONES}

ESTUDIOS

Comunicación y controversias sociocientificas en salud y medio ambiente

ISSN: 1856-9536

Doi: https://doi.org/10.12804/revistas.urosario.edu.co/disertaciones/a.10437

Volumen 14, Número 2 / Julio-diciembre 2021

Versión PDF para imprimir desde

http://revistas.urosario.edu.co/index.php/disertaciones

"[La energía nuclear] no solo necesita un enorme capital de instalación y altos costos de mantenimiento. También necesita asignaciones secretas, seguros patrocinados por el Estado y otros mecanismos perversos que son esenciales para la existencia de esta fuente" (CDP, 2011b).

El léxico utilizado y los argumentos empleados adquieren una virulencia particular, pero sin explicar realmente por qué. El enunciador no muestra la relación entre el proyecto HidroAysén y la energía nuclear, sino que la desprecia.

A partir del análisis se puede observar que los opositores, al igual que la empresa, también fueron adaptando su estrategia comunicacional a medida que avanzó el conflicto; sin embargo, su flexibilidad se adaptó mucho mejor a la coyuntura. No solo fueron capaces de integrar temas emergentes sensibles al momento sociopolítico, sino que lograron resignificar los argumentos de la propia empresa, por ejemplo, pasar de una postura de absoluta santuarización de la Patagonia a plantear como alternativa el desarrollo de energías renovables no convencionales. Además, en los insertos publicitarios analizados se observa una heterogeneidad de voces, entre ellas, destaca la de científicos y expertos que participan directamente en el debate, tanto individual como institucionalmente.

\section{Conclusiones}

Las producciones analizadas de los actores de esta controversia nutren un espacio mediático, el cual solamente es objeto de este estudio en la medida de que es la arena donde se desarrolla el debate público. Así, ese debate es testigo de que la producción de conocimientos y la movilización de argumentos científicos son elementos regulares y heterogéneos en la controversia sobre la instalación del Proyecto Hidroeléctrico Aysén.

En cuanto a la forma como los promotores del proyecto despliegan estos argumentos, se pueden identificar dos momentos en su estrategia comunicacional. En la primera parte, cuando el conflicto no se ha mediatizado y se inician los estudios preliminares, la empresa recluta a universidades y centros de investigación, utilizados como mecanismos de legitimación del EIA. También se aprecia cómo hacia el final de esta primera etapa la empresa desarrolla un dispositivo de responsabilidad social empresarial (donaciones, obras públicas, becas, comunicación con los habitantes, etc.). En una segunda instancia, y a medida que se mediatiza el conflicto, la empresa comienza a adaptar su estrategia comunicacional, dando respuesta a los argumentos de los opositores, sin renunciar a la construcción discursiva científico-técnica. Esta adaptación obligó a la empresa a un cambio de escala, que se vio reflejada en una gran campaña publicitaria nacional.

En cuanto a los opositores, nuestro análisis también arroja dos momentos clave en su estrategia comunicacional. En un primer momento, se centró en los habitantes de la región y se movilizaron argumentos en torno a la santuarización de la naturaleza y, posteriormente, se registró un cambio de escala que fue de lo local a lo nacional. Sin embargo, en este caso se observa una mayor heterogeneidad de argumentos y una mayor plasticidad discursiva. Por ejemplo, el CDP abraza tempranamente la idea del desarrollo sustentable, promueve el desarrollo de energías limpias y apela la participación ciudadana como valor fundamental para una democracia. Argumentos que, además, eran acordes al momento sociopolítico que vivía el país.

Así, observamos que en el desarrollo de esta controversia existen similitudes en las estrategias utilizadas por los actores, pero también diferencias fundamentales. En el caso de los argumentos científico-técnicos movilizados por los promotores, vemos cómo a medida que avanza el conflicto, la empresa moviliza argumentos de los 


\section{DISERTACIONES}

ESTUDIOS

expertos, pero estos dejan de aparecer de modo directo. Este distanciamiento no se observa en el caso de los opositores, en el cual podemos rastrear una participación directa de científicos y expertos, tanto individual como institucionalmente.

Nuestro análisis muestra que mientras los opositores configuraron una arquitectura abierta a la participación autónoma de diversos actores, la empresa configuró un posicionamiento cerrado, que se constituyó como un punto de pasaje obligado (Callon, 1984) respecto a la validación de los argumentos que sustentaban el proyecto. Esto se verifica en la conformación de la Comisión Asesora de Desarrollo Eléctrico (Ministerio de Energía, 2011), cuyas recomendaciones se alinearon claramente con el proyecto.

El resultado histórico de la controversia, que tuvo como consecuencia la anulación del proyecto y un inédito desarrollo de fuentes de energía no convencionales en Chile, no solamente tuvo como una de sus principales causas la oposición popular a la iniciativa, sino que también una embrionaria organización del mundo científico. De esta forma, se puede observar que en la última década se ha visto robustecida la participación de los científicos y expertos en el debate público.

\section{Referencias}

1. Acot, P. (1988). L'invention de l'écologie. PUF.

2. Adenda n. $^{\circ} 1$ en respuesta a la Solicitud de Aclaraciones, Rectificaciones o Ampliaciones a la Adenda del Proyecto "Proyecto Hidroeléctrico Aysén”. (2008). https://infofirma.sea.gob.cl/DocumentossEA/Mostrar Documento?docld=26/5a/38225374c8914af08067b51e062ebad99e93

3. Akrich, M., Callon, M., \& Latour, B. (2006). Sociologie de la traduction. Presses des Mines.

4. Arenas, F., \& Osses, P. (2012). Hydropower in Chilean Patagonia: Evaluating socio-economic conditions for resettlement and/or compensation of rural inhabitants. En R. Stimson \& K. E. Haynes (Eds.), Studies in Applied geography and spatial analysis: Addressing real world issues (pp. 346-357). Edward Elgar.

5. Aspe, Ch., \& Jacqué, M. (2012). Environnement et société: Une analyse sociologique de la question environnementale. Quae.

6. Baya-Laffite, N. (2016). Black-boxing sustainable development: Environmental impact assessment on the river Uruguay. En J.-P. Voß. \& R. Freeman (Eds.). Knowing governance: The epistemic construction of political order. Palgrave.

7. Barandarian, J. (2018). Science and environment in Chile: The politics of expert advice in a neoliberal democracy. MIT Press.

8. Beck, U. (2001). La société du risque: Sur la voie d'une autre modernité. Aubier.

9. Badouard, R., \& Mabi, C. (2015). Introduction. Hermès. La Revue, 73(3), 11-14.

10. Breton, P. (2000). La parole manipulée. La Découverte.

11. Broitman, C. (2017a). Entre la construction du point de vue et l'immersion sensible: comprendre le cours d'un projet. Le cas d'un projet de barrages hydroélectriques au Chili [tesis de doctorado, París Sorbonne/Paris 4].

12. Broitman, C. (2017b). Quand les voix se répondent. Questions de communication, (2), 29-50. 


\section{DISERTACIONES}

ESTUDIOS

Comunicación y controversias sociocientificas en salud y medio ambiente

ISSN: $1856-9536$

Doi: https://doi.org/10.12804/revistas.urosario.edu.co/disertaciones/a.10437

Volumen 14, Número 2 / Julio-diciembre 2021

Versión PDF para imprimir desde

http://revistas.urosario.edu.co/index.php/disertaciones

13. Brüggemann, M., Lörcher, L., \& Walter, S. (2020). Post-normal science communication: Exploring the blurring boundaries of science and journalism. Journal of Science Communication, 19(3). https://doi. org/10.22323/2.19030202

14. Buletti, N., \& Ejderyan, O. (2020). When experts feel threatened: Strategies of depoliticisation inparticipatory river restoration project. Area, (53), 151-160.

15. Callon, M. (1984). Some elements of a sociology of translation: Domestication of the scallops and the fishermen of St Brieuc Bay. The Sociological Review, 32(1 suppl), 196-233.

16. Carson, R. (1962). Silent Spring. Houghton Mifflin.

17. Charvolin, F. (1993). L'invention de l'environnement en France (1960-1971). Les pratiques documentaires d'agrégation à l'origine du ministère de protection de la nature et de l'environnement [tesis de doctorado, Universidad Pierre Mendès-France de Grenoble y École nationale supérieure de mines de Paris].

18. Chateauraynaud, F. (2011). Argumenter dans un champ de forces: Essai de balistique sociologique. Petra.

19. Collins, H. M., \& Evans, R. (2002). The third wave of science studies: Studies of expertise and experience. Social Studies of Science, 32(2), 235-296.

20. Collins, H. M., Evans, R., \& Weinel, M. (2017). sts as science or politics? Social Studies of Science, 47(4), 580-586.

21. Consejo de Defensa de la Patagonia (CDP). (2011a). El maquillaje verde de HidroAysén: La realidad frente a la propaganda [compilado de insertos publicitarios].

22. Consejo de Defensa de la Patagonia (CDP). (2011b). Chile necesita una revolución energética ahora [compilado de insertos publicitarios].

23. Cuevas, A. (2008). Conocimiento científico, ciudadanía y democracia. Revista Iberoamericana de Ciencia, Tecnología y Sociedad, 4(10), 67-83. http://www.scielo.org.ar/scielo.php?pid=\$1850-00132008000100006\& script=sci_arttext

24. Dussaillant, A., Benito, G., Buytaert, W., Carling, P., Meier, C., \& Espinoza, F. (2010). Repeated glacial-lake outburst floods in Patagonia: An increasing hazard? Nat Hazards, 54, 469-481. https://doi.org/10.1007/ s11069-009-9479-8

25. Dunlap, R. E., \& Catton, W. R. Jr. (1979). Environmental sociology. Annual Review of Sociology, 5, 243-273.

26. Dunlap, R. E. (2015). Environmental sociology. En International encyclopedia of the social \& behavioral sciences (deuxième édition), 7.

27. Feng, L., Wu, Q., Wu, W., \& Liao, W. (2020). Decision-maker-oriented vs. collaboration: China's public participation in environmental decision-making. Sustainability, 12(4), 1334. https://doi.org/10.3390/su12041334

28. García Palacios, E., González Galbarate, J. C., López Cerezo, J. A., Lujan, J. L., Martín Gordillo, M., Osorio, C., \& Valdés, C. (2001). Ciencia, tecnología y sociedad: Una aproximación conceptual. oв//BibliotecaNueva.

29. Goodwin, P., Jorde, K., Meier, C., \& Parra, O. (2006). Minimizing environmental impacts of hydropower development: Transferring lessons from past projects to a proposed strategy for Chile. Journal of Hydroinformatics, 8(4), 253-270. https://doi.org/10.2166/hydro.2006.005

30. Hannigan, J. (2006). Environmental sociology: A social constructivist perspective. Routledge \& Kegan Paul Books. 


\section{DISERTACIONES}

ESTUDIOS

Comunicación y controversias sociocientificas en salud y medio ambiente

ISSN: 1856-9536

Doi: https://doi.org/10.12804/revistas.urosario.edu.co/disertaciones/a.10437

Volumen 14, Número 2 / Julio-diciembre 2021

Versión PDF para imprimir desde

http://revistas.urosario.edu.co/index.php/disertaciones

31. Hernández, B., \& Huepe, C. (2019). Participación ciudadana en políticas públicas de energía: Reflexiones para un Chile energéticamente sustentable. Polis, 18(53). http://dx.doi.org/10.32735/s0718-6568/2019-n53-1390

32. HidroAysén. (2008). Estudio de impacto ambiental "Proyecto Hidroeléctrico Aysén". https://infofirma.sea. gob.cl/Documentossea/MostrarDocumento?docld=e8/03/92eaee0642bbba32b4c1a28aeec61da6e66d

33. HidroAysén. (2009). Aysén con energía. Boletín (9).

34. Informe Consolidado de Solicitud de Aclaraciones, Rectificaciones y/o Ampliaciones a el Estudio de Impacto Ambiental del Proyecto "Proyecto Hidroeléctrico Aysén (ICSARA). (2008). https://infofirma.sea.gob.cl/ Documentossea/MostrarDocumento?docld=69/07/768edff65ab16c21c12acdcaae73c1bcfd35

35. Jasanoff, S. (2009). The fifth branch: Science advisers as policymakers. Harvard University Press.

36. Krieg-Planque, A., \& Oger, C. (2010). Discours institutionnels: Perspectives pour les sciences de la communication. Mots. Les langages du politique, 94, 91-96.

37. Lezaun, J., \& Soneryd, L. (2007). Consulting citizens: Technologies of elicitation and the mobility of publics. Public Understanding of Science, 16(3), 279-297.

38. López Cerezo, J. A., \& Luján, J. L. (2000). Ciencia y política del riesgo. Alianza.

39. Meier, C. (2011). Hidroelectricidad realmente sustentable para Chile. https://verdeseo.files.wordpress. com/2011/05/hidroelectricidad-realmente-sustentable-cmeier2.pdf

40. Ministerio de Energía. (2011). Comisión Asesora de Desarrollo Eléctrico CADE [informe]. https://comisiondeenergiacichile.files.wordpress.com/2011/11/informe-cade-2011-11.pdf

41. Piper, F., \& Fajardo, A. (2011a). "Does Patagonia need dams?". E-letter respuesta a Vince, G., "A Craving for Hydropower". Science, 329(5990), 38.

42. Piper, F., \& Fajardo, A., (2011b), Dams and afforestation plans in Chilean Patagonia. Frontiers in Ecology and the Environment, 9(2), 96.

43. Ramírez, C., Sandoval, V., San Martín, C., Álvarez, M., Pérez, Y., \& Novoa, C. (2012). El paisaje rural antropogénico de Aisén, Chile: Estructura y dinámica de la vegetación. Gayana Botánica, 69(2), 219-231.

44. Ramos Torre, R. (1999). Prometeo y las Flores del Mal. En R. Ramos Torres \& F. García Selgas (Eds.), Globalización, riesgo y reflexividad: Tres temas de la teoría social contemporánea (pp. 249-274). Centro de Investigaciones Sociológicas.

45. Rocamora, V., Jara, R., \& Broitman Rojas, C. (2019). Cómo los expertos entienden al público en las controversias sobre vacunación en Chile. Revista Arbor, 195(794), a530.

46. Román, R. (2011). Diez razones por las cuales HidroAysén no es necesario, ni conveniente para Chile. http:// tostost.blogspot.com/2011/05/diez-razones-por-las-cuales-hidroaysen.html

47. Sannazzaro, J. (2011). Controversias científico-públicas: El caso del conflicto por las "papeleras" entre Argentina y Uruguay y la participación ciudadana. Revista Iberoamericana de Ciencia, Tecnología y Sociedad-CTS, 6(17), 213-239.

48. Tecklin, D., Bauer C., \& Prieto, M. (2011). Making environmental law for the market: The emergence, character, and implications of Chile's environmental regime. Environmental Politics, 20(6), 879-898.

49. Tironi, M., \& Barandiarán, J. (2014). Neoliberalism as political technology: Expertise, energy and democracy in Chile. En E. Medina, I. Costa Márques \& C. Holmes (Eds.), Beyond imported magic: Studying science and technology in Latin America. MIт Press. 


\section{DISERTACIONES}

50. Venturini, T. (2010). Diving in magma: How to explore controversies using actor-network theory. Public Understanding of Science, 19(3), 258-273. https://doi.org/10.1177/0963662509102694

51. Venturini, T., Ricci, D., Mauri, M., Kimbell, L., \& Meunier, A. (2015). Designing controversies and their publics. Design Issues, 31(3), 74-87.

52. Verón, E. (1978). Sémiosis de l'idéologie et du pouvoir. Communications (28), 7-20.

53. Verón, E. (1981). Construire l'événement: Les médias et l'accident de Three Mile Island. Minuit.

54. World Commission on Dams. (2000). Dams and development: A new framework for decision-making: The report of the world commission on dams. Earthscan.

55. Winemiller, K. O., McIntyre, P. B., Castello, L., Fluet-Chouinard, E., Giarrizzo, T., Nam, S., ... \& Sáenz, L. (2016). Balancing hydropower and biodiversity in the Amazon, Congo, and Mekong. Science, 351(6269), 128-129.

56. Wright, S. J., Habit, E., Adlerstein, S., Parra, O., \& Semrau, J. D. (2009). Graham Scholars Program: Sustainable education through an interdisciplinary international case study. Education for Sustainable Development, (4), 29-36. https://doi.org/10.1007/s11625-009-0067-3

57. Wright, S. J., Parra, O., \& Bledsoe, B. P. (2008). Give and take: The toll on ecosystems. Documento procedente de Sigma Xi's Critical Issues in Science Series 2008 "Year of Water".

58. Young, N., \& Matthews, R. (2007). Experts' understanding of the public: Knowledge control in a risk controversy. Public Understanding of Science, (16), 123-144. https://doi.org/10.1177/0963662507060586 